\title{
Contests and the Private Production of Public Goods
}

\author{
Martin Kolmar* and Andreas Wagener†
}

\begin{abstract}
The private provision of public goods generally suffers from two types of efficiency failures: sorting problems (the wrong individuals contribute) and quantity problems (an inefficient amount is provided). Embedding the provision game into a contest that rewards larger contributions with higher probabilities of winning a prize may remedy such failures. Applications include tenure decisions at universities, electoral competition among politicians, etc. We identify a tradeoff between the value of the prize and the decisiveness of the contest. High-powered incentives in contests may cause an overprovision of the public good or wasteful participation of unproductive individuals in the contest.
\end{abstract}

\section{JEL Classification: D74, H41, H70, K42}

\section{Introduction}

Academic research serves (at least) two functions. First, it adds to a society's pool of knowledge, which is a public good. Second, for the scientist herself, research output is a performance indicator that counts in the competition within the science community for academic positions, grants, prizes, and other largely private benefits. This double function of scientific research indicates that organizationally, academia links two mechanisms: the private provision of a public good (scientific knowledge is produced by individual research contributions) and a contest for private rents (academic positions are assigned to those with better research performance).

The implications of combining the private provision of public goods with a contest are the topic of this article. When non-excludable public goods are provided through private contributions, free-riding behavior and positive externalities typically lead to inefficient provision levels (see Bergstrom, Blume, and Varian 1986). Adding a contest in which each contribution to the public good enhances the chances for the contributing individual to win a certain prize may alleviate such failures. Contests add a mechanism that generally induces too much investment to an allocation problem that is plagued by too little investment. In standard games of public-goods provision, incentives to contribute to a public good are too weak from a social perspective. By contrast, in a contest in which each individual undertakes effort that increases his probability of winning a certain prize, each player's contribution reduces the

* Institute for Public Finance and Financial Law, University of St. Gallen, Varnbüelstrasse 19, CH-9000 St. Gallen, Switzerland; E-mail martin.kolmar@unisg.ch; corresponding author.

$\dagger$ University of Hannover, Department of Economics and Management, Koenigsworther Platz 1, D-30167 Hannover, Germany; E-mail wagener@sopo.uni-hannover.de.

Received October 2010; accepted August 2011. 
winning probability of the other agents. This generates too strong incentives to undertake (wasteful) effort.

By balancing positive externalities, contests can be beneficial in connection with the private provision of public goods: The prospect of winning a prize spurs individual incentives to contribute, raising the output of the public good. More technically, the positive externality inherent in making contributions to public goods may be internalized by an adequately designed contest if the marginal return of the contest is set equal to the marginal externality existing in a decentralized contribution game.

Without referring to contests this idea of counterbalancing externalities in public-goods games was already suggested by Cornes and Sandler (1984, 1994). Morgan (2000) provides the first article to present an example for compensating externalities in the form of lotteries, which can be understood as a special type of "contests as a means to finance public goods": Individual efforts in the contest are tantamount to buying lottery tickets, the probability of winning the prize increases proportionally to the amount of tickets bought, and lottery revenues are used to finance both the prize and a public good. Lotteries never achieve the first-best level of the public good, but asymptotic efficiency (if the population is "large") can be obtained (Morgan 2000). ${ }^{1}$

Contests - as discussed here-generalize lotteries in several crucial respects. Most importantly, they allow for individuals who differ in their productivities in the provision and, possibly, also in their valuation of the public good. The assumption of heterogeneity in productivities is not only empirically plausible (recall the example of academic research) but also conceptually important. This can be seen when two different aspects in the provision of public goods are distinguished. First, provision may be restricted to the financing of public goods, implicitly assuming that the production of the public good can, at zero delegation costs, be left to some agency in the background of the model. We call this problem the quantity problem of public-goods provision, and a contest in such situations can be viewed as a lottery (Morgan 2000; Maeda 2008). ${ }^{2}$ Monetary contributions have identical (marginal) productivities, regardless of who makes them. Second, for important public goods (think of scientific knowledge again), provision and production cannot be separated (i.e., the identity of the contributor matters with regard to the impact of a contribution). Specifically, individuals may differ in their abilities to produce the public good. This adds a sorting problem to the efficient provision of public goods: Only the 'right' individuals should be provided with incentives to contribute, and efficiency requires not only that a certain first-best quantity of the public good is provided but also that it is produced at the lowest possible opportunity costs. Thus, unlike a lottery, a public-goods contest tackles both the quantity and the sorting problems in publicgoods provision at the same time.

Our main observations in this article are as follows: With individual differences in productivities, an efficient sorting requires that (only) highly productive individuals devote all their effort to the public good, while individuals with low productivity specialize in the private good. To induce such an outcome, contest success functions must, on the one hand, give

\footnotetext{
${ }^{1}$ There are various ways around this inefficiency. Amegashie and Myers (2003) trace it back to the proportionality of the lottery technology and suggest convex raffles. Goeree et al. (2005) construct lowest-bid all-pay auctions, in which the highest bidder for sure wins the prize, and they show that such highly decisive contests yield higher revenues than do standard lotteries.

${ }^{2}$ The set-up in Maeda (2008) starts out with heterogeneous individuals. The main results of that article are, however, only derived for the case of identical individuals.
} 
high-powered (marginal) incentives to productive individuals to get them to specialize in publicgood provision but, on the other hand, must deter individuals at the lower end of the productivity distribution from contributing to the public good. ${ }^{3}$ If individuals have identical productivity in their contributions to the public good, there exists a continuum of efficient contests with "contest-success" functions à la Tullock (1980). The continuum is characterized by a negative relationship between the decisiveness and the prize of the contest, given that a minimum decisiveness is exceeded. Incentives for over- or under-investment result for contests with higher prizes and/or decisiveness and contests with lower prizes and/or decisiveness. If individuals differ with respect to their productivities of contributing to the public good, the class of Tullock contests that implement an efficient allocation is restricted to those with low prizes (to deter unproductive individuals) and high decisiveness (to provide high-powered incentives to productive individuals).

Combining the private provision of public goods with a contest has, in addition to lottery cases, several potential applications, ranging from incentives in teams (when performance of a team is measured by aggregate team output) to TV competitions such as American Idol or Britain's Got Talent, in which contestants provide a public good "entertainment" for the audience while aspiring to fame when winning the competition (Amegashie 2009). For illustration, consider the following important applications.

\section{Tenure Contests}

Career incentives for scientists are often organized as contests: There are a limited number of tenured positions or research grants to which a (usually much larger) number of scientists apply. A major selection criterion is typically the number and quality of publications. In such a contest, a publication record is only "good" as long as there is no one else with a better record, and if all scientists publish twice as much the odds in the competition for tenured jobs are more or less unchanged. Research efforts, however, also contribute to the provision of the public good "scientific knowledge." In the provision of knowledge, it is not primarily a fundraising problem that has to be solved but rather the sorting problem of how to motivate (only) individuals with a comparative advantage to do research. Without any private gains from research, scientific knowledge will likely remain undersupplied. Exposing scientists to contests in which they strive to win a prize (say, obtaining tenure) may sharpen their incentives, thus boosting the production of scientific knowledge.

To add contests to the production of knowledge goods (called "innovations") has, for example, been considered by Che and Gale (2003). However, unlike in our model, an innovation in this approach is not a public good. Maurer and Scotchmer (2004) discuss incentive mechanisms to elicit investments in new knowledge, understood as a public good. They emphasize the role of research contests as an alternative to intellectual property rights. Awarding prizes for research and afterwards putting the findings in the public domain avoids the inefficient exclusion of potential users of these findings that goes along with the assignment of intellectual property rights.

\footnotetext{
${ }^{3}$ Lange, List, and Price (2007) experimentally show that the set of contributors to a public good is larger with lotteries than in a voluntary-contribution game. Hence, incentives to participate play a crucial role in contest settings (although for the pure lottery case with equal productivities no sorting problem arises).
} 


\section{Electoral Competition among Politicians}

Ideally, politicians are supposed to promote social well-being, which-however one defines it - comes as a public good in many of its dimensions. Politicians can spend effort in various ways: generating inventive solutions, setting up political programs, rendering programs into implementable policies, implementing and communicating their policies, and so forth. Social well-being will be higher the greater the politicians' efforts. Yet (potential) politicians have an incentive to 'free-ride' on their colleagues' efforts, leaving societal problems treated only insufficiently. In democratic regimes, however, elections may offset this tendency toward carelessness and laxness. They put politicians in a contest in which more fruitful efforts (from the viewpoint of the populace) are rewarded with higher vote shares. For politicians, getting elected means power, prestige, satisfaction, and possibly riches. The efforts to win these "prizes" are contributions to the public good "social welfare." Again, the sorting problem is most relevant: Only the politically most talented and productive individuals should become politicians.

The rest of this article proceeds as follows. Section 2 sets up a model in which a public good can be produced by individuals with different productivities and characterizes efficient allocations. Section 3 introduces contests and derives sufficient conditions for their efficient design. Section 4 discusses the limitations of contests, and section 5 concludes. Proofs are collected in the Appendices.

\section{The Model}

\subsection{Set-Up}

Consider an economy that consists of $n \geq 2$ individuals, indexed by $i=1, \ldots, n$. Each individual is endowed with $m>0$ units of time ${ }^{4}$ that can be spent on an activity, called "work," that results in the production of a private consumption good or on another activity, called "contributions," that increases the supply of a public good, provided that the productivity of the contribution is positive (see below). Denote with $y_{i}$ the time individual $i$ spends on contributing to the public good. The rest of the time endowment $m-y_{i}$ goes into the production of the private good. In addition, each individual has an exogenous endowment $x^{e}>0$ of the private good. Later on we will introduce a tax to finance the prize of the contest. A positive endowment $x^{e}$ makes sure that tax revenues, and therefore the contest prize, can always be guaranteed to be positive, which ensures contest participation. ${ }^{5}$

Average and marginal productivities in the production of the private good are assumed to be constant, exogenous, and equal across individuals; we denote them by $w$. In a competitive setting, $w$ reflects the wage that can be earned through work or, more precisely, the wage differential between private- and public-goods production (where we have normalized the

\footnotetext{
${ }^{4}$ All individuals have the same (time) endowment. For a discussion of the role of endowments and their heterogeneity in (lottery) contests see Faravelli (2011).

${ }^{5}$ We have set up the sorting problem in the model to be presented below in the most extreme way as to allow for individuals that are full-time contributors. In the absence of type-specific taxes those types could not be taxed, and therefore the whole contest mechanism would not be able to fly. Alternatives to this assumption would be either personalized taxes (only partial or non-contributors get taxed, a type of discrimination that should be verifiable) or the enforcement of interior solutions that guarantee positive tax rates but that come at the price of limited generality.
} 
nominal wage of public-goods production to zero). Individual $i$ 's gross income amounts to $w \cdot\left(m-y_{i}\right)+x^{e}$.

The total output of the public good depends in a deterministic and nondecreasing way on individual contributions. More specifically, we assume that each individual $i$ is characterized by an innate productivity $r_{i} \geq 0$, which measures the marginal and average effect his contributions have on the output level of the public good; $r_{i} \cdot y_{i}$ is the amount that individual $i$ 's contribution adds to the public good. Hence,

$$
g=g(\mathbf{y})=\sum_{i=1}^{n} r_{i} \cdot y_{i}
$$

denotes the provision level of the public good. Here and subsequently, we denote vectors with boldface letters (e.g., $\mathbf{y}=\left(y_{1}, \ldots, y_{n}\right)$ ). As usual, the notation $\mathbf{y}_{-i}$ denotes the $n-1$ dimensional vector that contains all $y_{j}$ except for $y_{i}$. While we speak of the time $y_{i}$ that individual $i$ devotes to the provision of the public good as its "contribution," this does not exclude that the contribution may be meaningless in the sense that it may not increase the provision level of the public good $\left(r_{i}=0\right)$. Without loss of generality, we order individuals according to their productivities:

$$
r_{1} \geq r_{2} \geq \ldots \geq r_{n}
$$

Let $n_{i}$ be the number of individuals with productivity equal to that of $i$ 's; $n\left(r_{i}\right)=\#\left\{j \mid r_{j}=r_{i}\right\}$, where \# denotes the cardinality of a set. As we allow for zero productivities, there may exist $k \leq n$ such that $r_{i}=0$ for all $i \geq k$.

Each agent $i=1, \ldots, n$ has quasi-linear preferences over his consumption levels of the private $\left(x_{i}\right)$ and of the public good $(g)$,

$$
u_{i}\left(x_{i}, g\right)=x_{i}+v_{i}(g)
$$

All functions $v_{i}$ are increasing and strictly concave $\left(v_{i}{ }^{\prime}(g)>0>v_{i}{ }^{\prime \prime}(g)\right)$. To exclude uninteresting corner solutions, we assume that $v_{i}{ }^{\prime}(0)>1$ for all $i$ and $\sum_{i=1}^{n} v_{i}{ }^{\prime}\left(\sum_{j} r_{j} m\right)<w / r_{1}$. The first assumption ensures that not all resources ought to be devoted to private consumption, while the latter precludes that all resources should optimally be used to produce the public good.

While individual time endowments are clearly not transferable across individuals, the private good is. Therefore, an allocation $(\mathbf{x}, \mathbf{y}) \in \mathbb{R}_{+}^{2 n}$ is feasible if none of the $y_{i}$ violates the individual time constraint and the total consumption of the private good does not exceed its production:

$$
0 \leq y_{i} \leq m \text { for all } i \text { and } \sum_{i=1}^{n} x_{i} \leq w \cdot\left(n m-\sum_{i=1}^{n} y_{i}\right)+n x^{e} .
$$

\section{Efficiency}

As a result of the quasi-linearity of preferences, our setting hosts an infinity of Paretoefficient allocations in which some individuals do not consume anything of the private good $\left(x_{i}=0\right.$ for some $i$ ). We do not view these allocations as particularly relevant and omit them 
from further consideration. The remaining set of Pareto-efficient allocations is characterized in Proposition 1.

Proposition 1. Consider a Pareto-efficient allocation in which everybody consumes a positive amount of the private good $\left(x_{i}>0\right.$ for all $\left.i\right)$.

1. There exists a threshold productivity level $r *$ below which individuals do not contribute to the public good $\left(y_{j}=0\right.$ for all $j$ with $\left.r_{j}<r^{*}\right)$ and above which individuals are fulltime contributors $\left(y_{j}=m\right.$ for all $j$ with $\left.r_{j}>r^{*}\right)$. Individuals with zero productivity never spend any time producing the public good $\left(r_{i}=0 \Rightarrow y_{i}=0\right)$.

2. There exists a unique efficient amount

$$
g^{*}=Y^{*}+m \cdot \sum_{r_{j}>r^{*}} r_{j}
$$

of the public good where $Y^{*}$ denotes the sum of contributions of individuals with productivity $r^{*}$.

(a) Either $0<Y^{*}<m \cdot n\left(r^{*}\right)$, and $g^{*}$ satisfies

$$
\sum_{j=1}^{n} v_{j}^{\prime}\left(g^{*}\right)=\frac{w}{r^{*}} .
$$

(b) Or $Y^{*}=m \cdot n\left(r^{*}\right)$, and $g^{*}$ satisfies

$$
\frac{w}{r_{i^{*}-1}}>\sum_{j=1}^{n} v_{j}\left(g^{*}\right) \geq \frac{w}{r^{*}},
$$

where $r_{i^{*-1}}$ denotes the next smaller productivity than $r^{*}$.

The proof of Proposition 1 is in Appendix A. The result states that (only) individuals with a certain minimum productivity should contribute to the public good; individuals with productivity lower than the threshold level and, of course, totally unproductive individuals should not contribute at all. Some individuals (those with exactly the threshold productivity) split their time between contribution to the public good and work. If there is more than one individual in this group, the quasi-linearity of utility functions implies that only the total contributions of this group are fixed, whereas individual contributions can be freely shifted among group members to reach different allocations on the Pareto frontier. Individuals with higher productivity levels should become full-time contributors. The strict separation between contributors and noncontributors is a consequence of the quasi-linearity of preferences and constant productivities; it fits well with the empirical pattern of complete specialization to one profession.

As a corollary, in an efficient allocation individuals with low productivity never spend more time on public good provision than do individuals with high productivity:

$$
r_{i}>r_{j} \Rightarrow y_{i} \geq y_{j}
$$

Equation 5 is the standard Samuelson condition, with the marginal rate of transformation being determined by the threshold productivity level $r^{*}$. Equation 6 is a modified Samuelson condition: As a result of discontinuities in the distribution of the $r_{i}$, the equality in Equation 5 might not be feasible. If Equation 6 applies, all individuals with productivity $r^{*}$ and above are full-time contributors to the public good (i.e., $g^{*}=m \cdot \sum_{r_{j} \geq r^{*}} r_{j}$ ), while individuals with lower productivity specialize in the production of the private good. 
Proposition 1 shows the two dimensions of the efficiency problem: the sorting problem ("Who should contribute how much?") and the quantity problem ("How much of the public good should be provided?"). The sorting problem arises from the heterogeneity in productivities.

We illustrate Proposition 1 with two examples that will reappear later.

\section{Example 1: Identical Preferences}

All $n$ individuals have identical utility functions $u\left(x_{i}, g\right)=x_{i}+v(g)$ for $i=1, \ldots, n$. There are two productivity types of agents: highly productive ones with productivity $r_{H}$ and less productive ones with $r_{L}<r_{H}$. Denote the number of individuals with high productivity by $h=$ $n\left(r_{H}\right)$; assume $h>1$. For $h=n$ we get the "lottery case" of identical individuals, but for $h<n$ the sorting problem will arise. Total output of the public good amounts to $g(\mathbf{y})=r_{H} \sum_{i=1}^{h} y_{i}+$ $r_{L} \sum_{i=h+1}^{n} y_{i}$. Efficient allocations satisfy

$$
-w+n \cdot r_{i} \cdot v^{\prime}(g) \leq 0 \quad \text { for } i=1, \ldots, n,
$$

with equality if $y_{i}>0$. If $m$ is large (more precisely, if $r_{L} v^{\prime}\left(h r_{H} m\right)<w / n$ ), optimality requires that only highly productive individuals contribute to the public good, while the others specialize in private-good production $\left(y_{L}=0\right)$. Then, the efficient amount of the public good emerges from Equation 5 as:

$$
g^{*}=v^{-1}\left(\frac{w}{n r_{H}}\right)
$$

\section{Example 2: Different Preferences}

Suppose there are $n=3$ individuals. Individuals 1 and 2 have productivity $r_{H}$ while individual 3 has lower productivity $r_{L}<r_{H}$. Individuals 1 and 2 have identical utility functions $u_{i}=x_{i}+\log g$ (for $i=1,2$ ), while individual 3 has utility function $u_{3}=x_{3}+\beta \cdot \log g$ where $\beta>$ 0 . Three efficiency regimes can be distinguished (see Appendix B). With small values of $\beta$, (only) the highly productive individuals contribute parts of (but not all) their time to the production of the public good, while the low-productivity individuals specialize in the production of the private good (Regime 1). Intermediate values of $\beta$ require the high-productivity individuals to fully specialize in the production of the public good and the others to specialize in private-good production (Regime 2). With high values of $\beta$, the highly productive individuals should fully specialize in the production of the public good, while the low-productivity individual devotes time to the production of both goods (Regime 3).

\section{Provision of Public Goods by Means of Contests}

\section{Contest}

A public authority awards a prize of fixed value $z$, measured in units of the private good $x$ and to be shared among the contributors to the public good. The value of the prize is 
independent of the contributions to the public good and known to every agent prior to his timeallocation choice. ${ }^{6}$

We assume that the government finances the prize by lump-sum taxes $t$ on each agent. ${ }^{7}$ Hence, $z=n t$ and $t \leq \min _{i}\left[w\left(m-y_{i}^{*}\right), x^{e}\right]=x^{e}$ (because some individuals will be full-time contributors in a first-best solution in general). In the following we denote the maximum sum of taxes by $\bar{z}$.

Individual chances to win the prize depend on all agents' contributions. Denote by $p_{i}(\mathbf{y})$ individual $i$ 's probability to win the prize if contributions are given by vector $\mathbf{y}$. All probabilities add up to 1:

$$
\sum_{i} p_{i}(\mathbf{y})=1 \quad \text { for all } \mathbf{y}
$$

A selection of functions $\left(p_{i}(\mathbf{y})\right)_{i=1, \ldots, n}$ that satisfies Equation 10 is simply called a contest success function (CSF).

Given a CSF, individual $i$ 's decision problem is as follows:

$$
\max _{0 \leq y_{i} \leq m}\left\{w \cdot\left(m-y_{i}\right)+x^{e}-t+p_{i}\left(y_{i}, \mathbf{y}_{-i}\right) \cdot z+v_{i}\left(\sum_{j=1}^{n} r_{j} y_{j}\right)\right\} .
$$

To ensure existence of Nash equilibria, we assume that every component $p_{i}(\mathbf{y})$ of a CSF is concave in $\mathbf{y}$. This given, the first order conditions (FOC) of individual optimization are also sufficient to yield a best reply.

If individual $i$ chooses to undertake non-zero contributions, it equates marginal opportunity costs (in terms of foregone consumption in the private good) and marginal benefits (i.e., potential consumption gains in the private good through the prize plus increased consumption of the public good):

$$
-w+z \cdot \frac{\partial p_{i}}{\partial y_{i}}+r_{i} \cdot v_{i}^{\prime}(g)-\lambda_{i}=0
$$

where $\lambda_{i}=0$ whenever $y_{i}<m$. Individual $i$ decides not to participate in the contest (i.e., $y_{i}=0$ is found to be optimal) if and only if

$$
-w+z \cdot \frac{\partial p_{i}\left(0, \mathbf{y}_{-i}\right)}{\partial y_{i}}+r_{i} \cdot v_{i}^{\prime}(g) \leq 0 .
$$

In a Nash equilibrium, the condition outlined in Equation 12 or Equation 13 must simultaneously be satisfied for all $i$. Since individual strategy sets are compact and convex and payoffs are continuous and (quasi-)concave, at least one Nash equilibrium exists.

\section{No Contest}

For sake of reference let us assume that $z=0$ (and, consequently, $t=0$ ). We are then back in the standard decentralized provision game for public goods (see Cornes and Sandler 1996). A Nash equilibrium $\mathbf{y}^{N}$ of this game (with attending $g^{N}$ ) is then characterized by

\footnotetext{
${ }^{6}$ In the case of scientific research, the prize can be thought of as the monetary equivalent of a tenured position at a university or a research award; in the case of a political contest it may represent the utility of being elected into a certain office.

${ }^{7}$ This assumption may sound extreme at first. However, for the problem of contest design it is without relevance because any type of tax distortion could be easily counterbalanced by an adequately adjusted contest mechanism.
} 


$$
-w+r_{i} v_{i}{ }^{\prime}\left(g^{N}\right)-\lambda_{i}^{N} \leq 0 \quad \text { and } \quad y_{i}^{N} \cdot\left(-w+r_{i} v_{i}{ }^{\prime}\left(g^{N}\right)-\lambda_{i}^{N}\right)=0,
$$

and

$$
y_{i}^{N}-m \leq 0 \quad \text { and } \quad \lambda_{i}^{N} \cdot\left(y_{i}^{N}-m\right)=0,
$$

with $\lambda_{i}$ as the Lagrangian multiplier associated with individual $i$ 's time constraint $(i=1, \ldots, n)$. As expected, Equation 14 in general does not meet the efficiency requirements of Proposition 1; neither the quantity nor the sorting problems are solved efficiently. Typically, the public good is underprovided relative to the first best. Sorting failures emerge if low-productivity individuals have a strong preference for the public good compared to high-productivity individuals.

This can be verified in Example 2 above. A complete characterization of Nash equilibria (see Appendix C) shows that for certain parameter values, the low-productivity individual will in fact contribute to the public good, although efficiency requires that he fully specializes in the production of the private good. Even worse, this person may make larger contributions than highly productive individuals and, in the extreme, may be the only person contributing in a Nash equilibrium - which should not happen from Proposition 1.

From Equation 14, individuals with zero productivity in the provision of the public good will never contribute - as is required by efficiency considerations.

\section{Efficient Contests}

Suppose that $\mathbf{y}^{*}=\left(y_{1}^{*}, \ldots, y_{n}^{*}\right)$ is a vector of efficient contributions with corresponding threshold productivity $r^{*}$ and output $g^{*}$. For simplicity, suppose that the efficient allocation is characterized by Equation 5. Comparing the efficiency requirement from Proposition 1 with Equations 12 and 13 leads to Proposition 2.

Proposition 2. A concave CSF that implements efficient contributions $\mathbf{y}^{*}$ has the following properties:

1. Individuals with productivity below $r^{*}$ do not have any incentive to contribute:

$$
z \cdot \frac{\partial p_{j}\left(0, \mathbf{y}_{-j}^{*}\right)}{\partial y_{j}} \leq w-r_{j} v_{j}{ }^{\prime}\left(g^{*}\right) \quad \text { for all } j \text { with } r_{j}<r^{*} .
$$

2. Individuals with productivity above $r^{*}$ become full-time contributors:

$$
z \cdot \frac{\partial p_{j}\left(m, \mathbf{y}_{-j}^{*}\right)}{\partial y_{j}} \geq w-r_{j} v_{j}^{\prime}\left(g^{*}\right) \text { for all } j \text { with } r_{j}>r^{*} .
$$

3. Individuals with a productivity of $r^{*}$ must be given the incentive to implement Equation 5:

$$
z \cdot \frac{\partial p_{j}\left(y_{j}^{*}, \mathbf{y}_{-j}^{*}\right)}{\partial y_{j}}=r^{*} \cdot \sum_{k \neq j} v_{k}{ }^{\prime}\left(g^{*}\right) \text { for all } j \text { with } r_{j}=r^{*} \text {. }
$$

Though technical, Proposition 2 carries some important messages for contest design. 


\section{Outcome-Based Contest Success Functions}

A simple way to keep untalented contributors away from the contest is to reward outcomes $r_{i} y_{i}$, rather than contributions $y_{i}$, to the public good. With a CSF of type

$$
p_{i}=\tilde{p}_{i}(\mathbf{r} \times \mathbf{y})
$$

individuals with $r_{i}=0$ face zero (marginal and absolute) probability of winning the contest, which avoids socially wasteful entry of this group into the contest; the condition outlined in Equation 16 will always be satisfied.

The property highlighted in Equation 19 is quite natural in settings in which contributions (understood as effort) cannot be observed while outcomes can. Consider, for example, the case of research: Tenure decisions are not based on the (unverifiable) time that individuals spent on research but on the observable number and quality of their publications. ${ }^{8}$

\section{High Decisiveness at the Upper End}

The condition outlined in Equation 17 implies that in the upper range of the productivity distribution the contest must (still) be sufficiently sharp as to trigger maximum effort from highly productive contributors.

\section{Balancing Externalities}

Item 3 in Proposition 2 provides a condition for individuals with the threshold productivity $r^{*}$ (i.e., the least productive individuals who still make contributions). The condition outlined in Equation 18 requires that the marginal benefit from investing in the contest (i.e., prize times marginal increase in its share) must, for the marginal individual, just be equal to the positive externality that this individual generates for the rest of the economy by making a contribution. This implies that the CSF must at least locally be strictly monotonic $\left(\partial p_{j}\left(\mathbf{y}^{*}\right) / \partial y_{j}>0\right)$.

\section{Decisiveness Versus Prize}

Given that its right-hand side (RHS) is a constant, the condition outlined in Equation 18 implies an inverse relationship between the decisiveness of the contest (represented by the magnitude of $\partial p_{j} / \partial y_{j}$ ) and the value of the prize $z$ : A less discriminating contest must promise a higher prize. If the contest designer wishes to minimize the total prize necessary to motivate the agents to contribute to the public good, he should design the rules that link contributions to awarding the prize as "sharp" as possible, leading to a high degree of decisiveness.

\section{Discrimination}

Suppose there are two individuals, each with identical productivity $r^{*}$ but with different preferences for the public good; the RHS in Equation 18 will differ for these individuals. Consequently, the LHSs cannot be equal either. There are basically two ways to achieve this: to

\footnotetext{
${ }^{8}$ Amegashie (2009)'s analysis of whether TV competitions like American Idol should be singing contests (i.e., outcomeoriented and based on expert judgments) or popularity contests (with TV voting by the audience, also allowing for untalented performers to win the competition) is also related to this issue.
} 
apply personalized first-order functions in the CSF when individuals with equal abilities shall make equal contributions (which may be infeasible because the contest designer would need information about individual productivities) or to have individuals with equal abilities making different contributions (such that the partial derivatives can differ).

\section{Complexity}

Proposition 2 establishes $n$ conditions (one for each individual) for an efficient contest, indicating that shaping contests efficiently may be a formidably complicated task. However, if all individuals have identical preferences for the public good (i.e., if $v_{i}^{\prime}(g)=v^{\prime}(g)$ for all $i$ ), the CSF can be constructed in a natural way:

$$
r_{i}>r_{j} \Rightarrow \frac{\partial p_{i}\left(y_{i}, \mathbf{y}_{-i}\right)}{\partial y_{i}}>\frac{\partial p_{j}\left(y_{j}, \mathbf{y}_{-j}\right)}{\partial y_{j}}
$$

whenever $\mathbf{y}_{-i}=\mathbf{y}_{-j}$ and $y_{i}=y_{j}$ for all $i, j$. That is, if individuals $i$ and $j$ make identical contributions, a marginal increase in contributions implies a larger gain for the more productive individual. An important implication of Equation 20 is that the values of $z \cdot \partial p_{j} / \partial y_{j}$ in Equations 16 and 18 increase while the term $w-r_{j} v^{\prime}\left(g^{*}\right)$ decreases with individual $j$ 's productivity $r_{j}$. Hence, when designing the contest, the condition outlined in Equation 16 must hold for the most productive group below $r^{*}$ (since then Eqn. 16 will also hold for less productive individuals), while the condition outlined in Equation 18 needs only be satisfied for the least productive group above $r^{*}$ (which then implies that it will hold for more productive individuals as well). Thus, Proposition 2 basically only consists of three, rather than $n$, conditions. This facilitates contest design considerably.

\section{Tullock Contests}

To illustrate the chances and limitations of contests in conjunction with the private provision of public goods, we employ the widely used Tullock CSF,

$$
p_{i}\left(y_{i}, \mathbf{y}_{-i}\right)=\frac{\left(r_{i} y_{i}\right)^{\alpha}}{\sum_{j=1}^{n}\left(r_{j} y_{j}\right)^{\alpha}} \quad(i=1, \ldots, n),
$$

where the parameter $\alpha>0$ determines the decisiveness of the contest (Tullock 1980; Skaperdas 1996). ${ }^{9}$ It measures the degree of competition generated by the contest rules (i.e., by the mapping of contributions into award decisions). For $\alpha \rightarrow 0$, the contest does not discriminate at all between agents $\left(p_{i} \rightarrow 1 / n\right)$, independent of their contributions, while for $\alpha \rightarrow \infty$, the contest awards the prize to the contestant with the highest $r_{i} y_{i}$ with probability 1 . Observe that Equation 21

\footnotetext{
${ }^{9}$ Observe that for $\alpha>1$ the Tullock CSF is not concave over the whole domain $[0, m]^{n}$. For $\alpha>1$ and $A=\sum_{j=1}^{n}\left(r_{j} y_{j}\right)^{\alpha}$, $p_{i}$ is convex for all $y_{i}<\{[(a-1) A] /(1+a)\}^{1 / a} / r_{i}$ and concave thereafter. While Proposition 2 was obtained for globally concave CSFs and optimality could be established by FOCs, this is no longer true for the general Tullock function. An additional problem of standard contests is that equilibria may not exist if $\alpha$ is too large; then interior solutions may violate individual participation constraints. This problem does not exist for our public-goods contest as a result of the intrinsic value of the investments $y$.
} 
determines success in the contest on the basis of output $r_{i} y_{i}$ rather than on input $y_{i}$. Moreover, the Tullock function outlined in Equation 21 satisfies the condition outlined in Equation 20.

\section{Identical Preferences-Example 1 (Continued)}

Let us reconsider the set-up with $h \geq 2$ high-productivity $\left(r_{H}\right)$ and $(n-h) \geq 0$ lowproductivity $\left(r_{L}\right)$ individuals. Without loss of generality, assume that $r_{H}=1>r_{L}$. We will focus on the case in which only high-productivity individuals should contribute $\left(r_{L} v^{\prime}\left(h r_{H} m\right)<w / n\right)$ because only in this case is the sorting problem relevant.

An efficient contest design for the high-productivity individuals requires, in this case (for a symmetric within-group Nash equilibrium), that

$$
\alpha \cdot z=\left[\frac{n-1}{h-1} \cdot \frac{h}{n}\right] \cdot w \cdot g^{*} .
$$

Equation 22 exhibits the reciprocal relationship between the decisiveness parameter $\alpha$ and the size of the prize $z$ : The contest is cheaper the more decisive it is, and vice versa. A contest with Tullock contest-success function never induces totally unproductive individuals to make contributions. ${ }^{10}$ Still, the contest may trigger an over-provision of the public good-if decisiveness and/or prize are chosen - such that $\alpha \cdot z$ exceeds the RHS of Equation 22.

The above solution is trivial to implement if $r_{L}=0$. The situation is more complex when $r_{L}>0$. In Appendix D we prove Proposition 3.

Proposition 3. Suppose that the economy consists of two types of individuals who have identical preferences but different productivities of their contributions to the public good.

1. Tullock contests with $\alpha<1$ cannot implement an efficient allocation.

2. A Tullock contest with $\alpha=1$ can implement an efficient allocation if and only if $r_{\mathrm{L}} \leq$ $n(h-1) /(n h-1)$.

3. It is possible to implement the efficient allocation through a Tullock contest with $\alpha>1$. However, this might require very small prizes, $z \rightarrow 0$, and extreme levels of decisiveness ${ }^{11} \alpha=\frac{(n-1) h}{(h-1) n} \frac{w g^{*}}{z}$.

Depending on the decisiveness parameter $\alpha$, the prospect of winning a prize may induce low-productivity individuals to make unwarranted contributions to the public good. Moreover, if the Tullock CSF is not sufficiently 'steep' in the range of high contributions it fails to motivate high-productivity individuals to make such contributions.

With a strictly concave Tullock function $(\alpha<1)$, these problems cannot be simultaneously solved (first item of Proposition 3). With $\alpha=1$, efficiency is possible if the low-productivity $r_{L}$ is small, relative to $n$ and to $h$ (second item of Proposition 3). This is intuitive: A relatively large

\footnotetext{
${ }^{10}$ If all productivities are identical $(h=n)$, the lottery case emerges. The condition outlined in Equation 22 then shows that Morgan's impossibility result (lotteries are only asymptotically efficient) can be overcome by allowing for $\alpha>1$. This could be implemented by nonlinear pricing for lottery tickets (decreasing marginal costs of ticket purchase; see also Amegashie and Myers [2003]).

${ }^{11}$ Observe that the proof of the proposition shows that it might be possible to implement the efficient allocation with contests that are far less decisive.
} 
$r_{L}$ implies that the comparative disadvantage of the low-productivity type in the contest is relatively small. When either the total number of individuals or the total number of high-productivity individuals is small, an investment in the contest has a relatively large impact on the probability to win. Hence, the sorting problem can only be efficiently solved if the productivity differential is sufficiently large and/or if the economy is large. Finally, for $\alpha>1$ the Tullock function is convex-concave. Hence, the aims of deterring the low-productivity individuals and encouraging the highly productive ones are in harmony with each other. The convexity of the Tullock function at small contribution levels implies, however, that the first-order conditions are no longer sufficient. It must be checked separately whether the utility of the low-productivity individuals from not contributing does not fall short of that from starting to do so. This will be the case if the prize is of small value only. Low-value prizes require, however, a high decisiveness in order to get incentives to contribute for the high-productivity individuals straight.

\section{Heterogeneous Preferences-Example 2 (Continued)}

In Example $2\left(n=3, r_{1}=r_{2}=r_{H}>r_{L}=r_{3}\right)$, the low-productivity individual also differs from the high-productivity ones based on his preference intensity, $\beta$. The most interesting out of the three different efficiency regimes (see section 3 and Appendix B) is Regime 1 (i.e., $\beta \leq$ $2(m w-1))$, where an efficient solution requires positive contributions of the highly productive individuals, $y_{1}+y_{2}=(2+\beta) / w$, and no contributions by the low-productivity individual, $y_{3}=$ 0 . We will henceforth assume that the contest aims at implementing a symmetric Nash equilibrium where $y_{1}=y_{2}=(2+\beta) /(2 w)$.

As in Example 1, the case $r_{L}=0$ is simple, and the sorting problem can always be solved efficiently with the help of a contest. For $r_{L}>0$, Appendix E proves the following analog of Proposition 3.

Proposition 4. Suppose that the economy consists of two high-skilled individuals and one low-skilled individual with different preferences for the public good. Suppose further that efficiency requires that only the highly productive individuals make contributions to the public good.

1. Tullock contests with $\alpha<1$ fail to implement efficient allocations.

2. Tullock contests with $\alpha=1$ solve the quantity and the sorting problem if and only if (i) productivity differences between individuals are large $\left(r_{H} / r_{L} \geq 3\right)$ or (ii) if $\beta<2\left(r_{H} / r_{L}-\right.$ 1) $/\left(3-r_{H} / r_{L}\right)$ and $r_{H} / r_{L}<3$.

3. It is possible to implement the efficient allocation through Tullock contests with $\alpha>1$. However, this might require very small prizes $z \rightarrow 0$ and high levels of decisiveness.

While the cases $\alpha \neq 1$ follow along the same lines as in Example 1, the case $\alpha=1$ offers a new insight. Clearly, low productivities are a natural deterrent from the contest. Hence, if the low-productivity individual is sufficiently unproductive, compared to $r_{H}$, he will never engage in the contest. In addition, a high preference for the public good by the low-productivity individual might create an incentive to invest in the contest. This generates a conflict of interest because the highly productive individuals need a sufficiently high prize in order to be willing to invest the efficient quantities, and this prize depends positively on the low-productivity individual's preferences. High prizes, however, increase the incentive for the low-productivity 
individual to contribute to the public good. This conflict of interest can only be resolved if the low-productivity individual's preference for the public good is not too strong.

\section{Concluding Remarks}

Contests are used in various real-world problems in which the provision of public goods is decentralized. They have the potential to improve efficiency, relative to a pure private-provision game. Appropriately constructed, they may even support a first-best allocation under specific circumstances.

When used as an incentive device in the provision of public goods, contests typically have to solve two types of problems: the quantity problem to implement the efficient quantity of the public good and the sorting problem to deter individuals from making contributions the opportunity costs of which are too high. We characterized the structure of the contest function to solve these problems.

The literature on lottery-financing of public goods (see Amegashie and Myers 2003) shows that in the absence of a sorting problem efficiency can be obtained with a wide class of Tullock CSFs. There exists an inverse relationship between the efficient contest prize and the sharpness parameter (as long as the decisiveness exceeds a minimum level implicitly defined by financing constraint). Our article shows that things are more complicated once a sorting problem arises - which is a pervasive ingredient in those many instances of public-goods provision in which the individual characteristics of the contributor matter. Deterring lowproductivity individuals from making unwarranted contributions to the public good requires a high level of decisiveness and a small total prize. While efficiency can still be reached, the range of efficient contests is restricted. Observations like this call for a careful design of contests in real-word situations as well as for more research on the efficiency properties of contests as incentive devices in general.

\section{Appendix A: Proof of Proposition 1}

By choice of non-negative $x_{i}$ and $y_{i}$, Pareto-efficient allocations maximize the utility of individual 1, subject to the constraints that all other individuals reach utility levels of at least $\bar{u}_{j}$ and that the allocation is feasible. The corresponding Kuhn-Tucker Lagrangian is

$$
\mathcal{L}(\mathrm{x}, \mathrm{y}, \lambda, \beta, \mu)=\sum_{i=1}^{n} \beta_{i} \cdot\left[x_{i}+v_{i}\left(\sum_{j=1}^{n} r_{j} y_{j}\right)-\bar{u}_{i}\right]-\sum_{i=1}^{n} \lambda_{i} \cdot\left[y_{i}-m\right]-\mu \cdot\left[\sum_{i=1}^{n} x_{i}-w \cdot\left(n \cdot m-n x^{e}-\sum_{i=1}^{n} y_{i}\right)\right] .
$$

For the sake of a lean notation we have included a multiplier $\beta_{1}$ for individual 1 in the objective function. The program outlined in Equation A. 1 is phrased such that all constraints are of type $h(\mathbf{x}, \mathbf{y}) \leq 0$ with linear or concave functions $h$. Hence, all multipliers have to be non-negative in an optimum. Moreover, $\beta_{1}=1$ will hold in an optimum. The (relevant) Kuhn-Tucker conditions for Equation A.1 are

$$
\begin{gathered}
\frac{\partial \mathcal{L}}{\partial y_{i}}=r_{i} \cdot \sum_{j=1}^{n} \beta_{j} v_{j}{ }^{\prime}(g)-\mu w-\lambda_{i} \leq 0 \wedge y_{i} \cdot \frac{\partial \mathcal{L}}{\partial y_{i}}=0, \\
\frac{\partial \mathcal{L}}{\partial x_{i}}=\beta_{i}-\mu \leq 0 \wedge x_{i} \cdot \frac{\partial \mathcal{L}}{\partial x_{i}}=0,
\end{gathered}
$$




$$
\frac{\partial \mathcal{L}}{\partial \lambda_{i}}=y_{i}-m \leq 0 \wedge \lambda_{i} \cdot \frac{\partial \mathcal{L}}{\partial \lambda_{i}}=0
$$

and $x_{i}, y_{i}, \lambda_{i} \geq 0$ for $i=1, \ldots, n$.

We characterize the optimal provision level of the public good as well as the optimal individual contributions to its production. We start with the latter.

It follows from our assumption on $v_{i}$ that $y_{i}<m$ for at least one $i$ (not all resources are devoted to the production of $g$ ). ${ }^{12}$ Moreover, $y_{i}>0$ for at least one $i$ with $r_{i}>0 .{ }^{13}$ Assume (as posited) that $x_{i}>0$ for all $i$. From Equation A.3,

$$
\beta_{i}=\mu=1 \text { for all } i,
$$

since $\beta_{1}=1$ (see above). ${ }^{14}$ This immediately implies from Equation A.2 that $y_{i}=0$ if $r_{i}=0$.

There exists at least one individual $i$ such that $y_{i}<m$. For this individual, $\lambda_{i}=0$ and, thus, from Equation A.2,

$$
r_{i} \cdot \sum_{j=1}^{n} v_{j}^{\prime}(g)-w=0 \quad \text { or } \quad y_{i}=0 .
$$

Suppose that $y_{i}>0$. Then the first equality in Equation A.6 holds. The same then applies to all other individuals $k$ with identical productivity $r_{k}=r_{i}$. For all individuals with higher productivity $r_{k}>r_{i}$ we have $r_{k} \cdot \sum_{j=1}^{n} v_{j}^{\prime}(g)-w>0$ such that $\lambda_{k}>0$ must hold from Equation A.2, and, therefore, $y_{k}=m \geq y_{i}$ from Equation A.4. For all individuals with lower productivity $r_{k}<r_{i}$ we get $r_{k} \cdot \sum_{j=1}^{n} v_{j}^{\prime}(g)-w-\lambda_{k} \leq r_{k} \cdot \sum_{j=1}^{n} v_{j}{ }^{\prime}(g)-w<0$, such that $y_{k}=0$ from Equation A.2. If $y_{i}$ $=0$ in Equation A.6, then $y_{j}=0$ for all $j$ with $r_{j} \leq r_{i}$.

To see the uniqueness of the optimal amount $g^{*}$ for an optimal set of contributors and their activities, assume to the contrary that there exists another $\tilde{g} \neq g^{*}$ such that Equations A.2-A.4 are fulfilled. Assume, for example, that $\tilde{g}=g^{*}$ $+\mathrm{d} g>g^{*}$. The case $\tilde{g}<g^{*}$ follows along similar lines.

An increase from $g^{*}$ to $\tilde{g}$ ceteris paribus has a positive utility effect of $v_{j}\left(g^{*}+\mathrm{d} g\right)-v_{j}\left(g^{*}\right)$ on each individual $\sum_{j=1, \ldots, n}^{n}$. Hence, in terms of the private good, ${ }^{15}$ the aggregate willingness-to-pay for a switch from $g^{*}$ to $\tilde{g}$ amounts to $\left(v_{j}\left(g^{*}+\mathrm{d} g\right)-v_{j}\left(g^{*}\right)\right)$.

Somebody has to increase their contributions in order to make the additional production $\mathrm{d} g$ possible. Assume that the threshold productivity for the provision of $g^{*}$ was $r^{*}$. Individuals with higher productivities $r>r^{*}$ cannot contribute to $\mathrm{d} g$ because they are full-time contributors already. Therefore, $\mathrm{d} g$ has to be delivered by individuals with productivities of $r^{*}$ or below.

1. Assume that $\mathrm{d} g$ can be produced by the group of individuals with productivity $r^{*}$ alone. Then, a total amount of additional contributions of $\sum_{r_{i}=r^{*}} \mathrm{~d} y_{i}=\mathrm{d} g / r^{*}$ is necessary from that group. In terms of the private good, individual $i$ 's opportunity costs for additional contributions $\mathrm{d} y_{i}$ are $w \times \mathrm{d} y_{i}$. Thus, the total amount of resources needed to compensate the $r^{*}$-group for their additional contributions adds up to $w \sum_{r_{i}=r^{*}} \mathrm{~d} y_{i}=w / r^{*} \cdot \mathrm{d} g$. The new allocation with $\tilde{g}$ can therefore only be Pareto-efficient if

$$
\sum_{j=1}^{n}\left(v_{j}\left(g^{*}+\mathrm{d} g\right)-v_{j}\left(g^{*}\right)\right) \geq w / r^{*} \times \mathrm{d} g .
$$

The strict concavity of the $v_{j}($.$) implies that v_{j}\left(g^{*}+\mathrm{d} g\right)-v_{j}\left(g^{*}\right)<v_{j}{ }^{\prime}\left(g^{*}\right) \mathrm{d} g$ for any $\mathrm{d} g>0$. Then, however, it must be true that

$$
\sum_{j=1}^{n}\left(v_{j}\left(g^{*}+\mathrm{d} g\right)-v_{j}\left(g^{*}\right)\right)<\sum_{j=1}^{n} v_{j}^{\prime}\left(g^{*}\right) \mathrm{d} g=w / r^{*} \cdot \mathrm{d} g,
$$

since $g^{*}$ is efficient. Hence, Equation A.7 can never be fulfilled for $\mathrm{d} g>0$.

\footnotetext{
${ }^{12}$ Suppose $y_{i}=m$ for all $i$. Consider a slight reduction of an arbitrary $y_{j}$. It increases production of $x$ by $w$ units while lowering $g$ by $r_{j}$ units. Since $\sum_{i=1}^{n} v_{i}{ }^{\prime}\left(\sum_{j} r_{j} m\right)<w / r_{1} \leq w / r_{i}$, the additional units of $x$ could be distributed across agents such that everyone is better off

${ }^{13}$ Suppose not (i.e., $g=0$ ). Since $v_{i}^{\prime}(0)>1$ for all $i$, a productive individual (with $r_{i}>0$ ) could start producing $g$, thereby increasing everybody's utility (including his own).

${ }^{14}$ Hence, as $\mu>0$, the feasibility constraint is binding: $\sum x_{i}=w\left(n m-\sum y_{i}\right)$. Moreover, from Equation A.5, the search for Pareto-efficient allocations with $x_{i}>0$ for all $i$ is tantamount to the maximization of the unweighted sum of utilities. None of the arguments for the set of active researchers and their contributions depends on the $\bar{u}_{i}$ in the KuhnTucker Lagrangian.

${ }^{15}$ We could also express compensations in terms of (additional) contributions $y$ to the public good. Both approaches are economically equivalent: An additional contribution $\mathrm{d} y_{i}$ has opportunity costs in terms of $x$ of $w \mathrm{~d} y_{i}$.
} 
2. Assume that $\mathrm{d} g$ has to be (partly) produced by individuals with "low" productivities $r_{i}<r^{*}$. Then, the sum of the additional contributions necessary to produce $\mathrm{d} g$ is larger than in case 1 above. So are its opportunity costs; they strictly exceed $w / r^{*} \mathrm{~d} g$. The condition outlined in Equation A.7 now holds even less frequently than before.

Hence, allocations other than $g^{*}$ (together with its efficient provision) cannot be Pareto-efficient. This proves uniqueness. $Q E D$.

\section{Appendix B: Efficient Allocations in Example 2}

Define $\rho:=r_{H} / r_{L}>1$.

REGIME 1. If $\beta \leq 2(m w-1)$, efficiency requires the Samuelson condition (Eqn. 5) to hold: $g^{*}=(2+\beta) r_{H} / w$. Only the highly productive individuals should contribute to the public good: $y_{1}+y_{2}=(2+\beta) / w$ and $y_{3}=0$.

REGIME 2. If $2(m w \rho-1) \geq \beta>2(m w-1)$, efficiency requires $g^{*}=2 m r_{H}$. The highly productive individuals are fulltime contributors, while individual 2 specializes in the production of the private good. The standard Samuelson condition (Eqn. 5) is violated, but the modified condition (Eqn. 6) holds: $w / r_{L} \geq(2+\beta) / g^{*}>w / r_{H}$.

Regime 3. If $\beta>2(m w \rho-1)$, efficiency requires $g^{*}=(2+\beta) r_{L} / w$ with $y_{1} *=y_{2} *=m$ and $y_{3} *=(2+\beta) / w-2 m \rho>0$ : Both types of individuals contribute to the public good; highly productive individuals contribute full time, the lowproductivity individual contributes part time. ${ }^{16}$ The efficient output $g^{*}$ of the public good satisfies the condition outlined in Equation 5: $(2+\beta) / g^{*}=w / r_{L}$.

\section{Appendix C: Nash Equilibria in Example 2}

First consider the optimal behavior of a high-productivity individual $i \in\{1,2\}$. Denoting the other highproductivity individual by $j$, individual $i$ maximizes, by choice of $y_{i}, u_{i}=w\left(m-y_{i}\right)+\log \left(r_{H} y_{i}+r_{H} y_{j}+r_{L} y_{3}\right)$, which gives rise to the following best-response function:

$$
y_{i}\left(y_{j}, y_{3}\right)= \begin{cases}m & \text { if } y_{j}+\frac{y_{3}}{\rho} \leq \frac{1}{w}-m \\ \frac{1}{w}-\left(y_{j}+\frac{y_{3}}{\rho}\right) & \text { if } \frac{1}{w}-m<y_{j}+\frac{y_{3}}{\rho} \leq \frac{1}{w} \\ 0 & \text { if } y_{j}+\frac{y_{3}}{\rho}>\frac{1}{w} .\end{cases}
$$

Nash equilibria can be described in terms of the total contributions of the high-productivity individuals; denote them by $Y_{H}:=y_{1}+y_{2}$. Combine the best-response functions of the high-productivity individuals to obtain that a Nash equilibrium must satisfy: ${ }^{17}$

$$
Y_{H}\left(y_{3}\right)= \begin{cases}2 m & \text { if } \frac{1}{w}-\frac{y_{3}}{\rho}>2 m \\ \frac{1}{w}-\frac{y_{3}}{\rho} & \text { if } 0 \leq \frac{1}{w}-\frac{y_{3}}{\rho} \leq 2 m \\ 0 & \text { if } \frac{1}{w}-\frac{y_{3}}{\rho}<0 .\end{cases}
$$

Individual 3 maximizes, by choice of $y_{3}, u_{3}=w\left(m-y_{3}\right)+\beta \log \left(r_{H} Y_{H}+r_{L} y_{3}\right)$. His best-response function is:

$$
y_{3}\left(Y_{H}\right)= \begin{cases}m & \text { if } Y_{H} \leq \frac{\frac{\beta}{w}-r_{L} m}{\rho} \\ \frac{\beta}{w}-\rho \times Y_{H} & \text { if } \frac{\frac{\beta}{w}-r_{L} m}{\rho}<Y_{H} \leq \frac{\beta}{\rho w} \\ 0 & \text { if } Y_{H}>\frac{\beta}{\rho w} .\end{cases}
$$

Functions $Y_{H}\left(y_{3}\right)$ and $y_{3}\left(Y_{H}\right)$ are parallel in $\left(Y_{H}, y_{3}\right)$-space. Distinguish two cases, as follows.

${ }^{16}$ If $\beta>w m(2 \rho+m)-2$, then $y_{i}=m$ for all $i$ would be optimal--which we have excluded from Proposition 1 .

${ }^{17}$ Clearly, $Y_{H}=2 m$ is only possible if $y_{1}=y_{2}=m$, and $Y_{H}=0$ is equivalent to $y_{1}=y_{2}=0$. 
1. First suppose that $m<1 /(2 w)$ (i.e., the case $Y_{H}=2 m$ can become relevant). Here the following subcases can be distinguished:

(a) $\beta \rho>1$. In all Nash equilibria, $Y_{H}^{N}=1 / w-m r_{L} / r_{H}$ and $y_{3}=m$. Hence, $g^{N}=r_{H} / w$.

(b) $\beta \rho=1$. In all Nash equilibria, $0<Y_{H}^{N}<2 m, 0<y_{3}^{N}<m$, and $g^{N}=r_{H} Y_{H}^{N}+r_{L} y_{3}^{N}=r_{H} / w$.

(c) $\beta \rho<1$.

(i) If $\beta \rho>2 m / w$, in all Nash equilibria $Y_{H}^{N}=2 m$ and $y_{3}^{N}=\beta / w-2 m r_{H} / r_{L}$. Hence, $g^{N}=r_{L} \beta / w$.

(ii) If $\beta \rho \leq 2 m / w$, in all Nash equilibria $Y_{H}^{N}=2 m$ and $y_{3}^{N}=0$. Hence, $g^{N}=2 m r_{H}$.

2. Now suppose that $m \geq 1 /(2 w)$. Hence, the case $Y_{H}=2 m$ cannot become relevant. Again, several subcases have to be distinguished, as follows.

(a) $\beta \rho>1$. For all Nash equilibria, $Y_{H}^{N}=\max \{0,1 / w-m / \rho\}$ and $y_{3}=\min \{m, \rho / w\}$. If $m>\rho / w$, the Nash equilibrium is unique with $\left(y_{1}^{N}, y_{2}^{N}, y_{3}^{N}\right)=(0,0, \rho / w)$. We get $g^{N}=r_{H} / w$ for all Nash equilibria.

(b) $\beta \rho=1$. For all Nash equilibria, $0<Y_{H}^{N}<1 / w$ and $0<y_{3}^{N}<\min \{m, \rho / w\}$, such that $g^{N}=r_{H} Y_{H}^{N}+r_{L} y_{3}^{N}=$ $r_{H} / w$.

(c) $\quad \beta \rho<1$. For all Nash equilibria, $Y_{H}^{N}=1 / w, y_{3}^{N}=0$, and $g^{N}=r_{H} / w$.

In summary, decentralized provision typically fails to solve the quantity problem: In most cases, the Nashequilibrium quantity of the public good amounts to $g^{N}=r_{H} / w$, which, from Appendix B, is inefficient. Neither is the sorting problem solved efficiently: For example, in many scenarios of case 2 above, the low-productivity individual 3 makes positive contributions. Even worse, in some cases above individual 3 (possibly) makes higher contributions than the highly productive individuals - which should never happen from Proposition $1 .{ }^{18}$

\section{Appendix D: Proof of Proposition 3}

The partial derivative of $p_{i}$ with respect to $y_{i}$ is

$$
\frac{\partial p_{i}\left(y_{i}, \mathbf{y}_{-i}\right)}{\partial y_{i}}=\alpha \cdot r_{i}^{\alpha} \cdot y_{i}^{\alpha-1} \cdot \frac{\sum_{j \neq i}\left(r_{j} y_{j}\right)^{\alpha}}{\left[\sum_{j=1}^{n}\left(r_{j} y_{j}\right)^{\alpha}\right]^{2}} .
$$

From Proposition 2 and the previous results for this set-up we know that, in an efficient allocation in which identical individuals behave identically, we must have

$$
\alpha \cdot r_{L}^{\alpha} \cdot 0^{\alpha-1} \cdot \frac{h \cdot\left(y_{H}^{*}\right)^{\alpha}}{h^{2} \cdot\left(y_{H}^{*}\right)^{2 \alpha}} \cdot z \leq w \cdot\left(1-\frac{r_{L}}{n}\right),
$$

and

$$
\alpha \cdot\left(y_{H}^{*}\right)^{\alpha-1} \cdot \frac{(h-1) \cdot\left(y_{H}^{*}\right)^{\alpha}}{h^{2} \cdot\left(y_{H}^{*}\right)^{2 \alpha}} \cdot z=w \cdot \frac{n-1}{n} .
$$

Here $y_{H}^{*}=g * / h$ is the efficient symmetric contribution of a high-productivity individual. The condition outlined in Equation D.2 guarantees that $y_{L}=0$, while the condition outlined in Equation D. 3 ensures that $y_{H}$ is at the efficient contribution level.

Depending on $\alpha$, we have to distinguish three cases.

Case 1: $\alpha<1$. Here, the Tullock function is strictly concave and it suffices to check marginal conditions to verify whether low-productivity individuals enter the contest. For $\alpha<1$, the first of these conditions can never be satisfied since $\lim _{y \rightarrow 0} y^{\alpha-1}=\infty$.

Case 2: $\alpha=1$. One can show that Equations D.2 and D.3 are consistent if

$$
r_{L} \leq \frac{(h-1) n}{h n-1}
$$

Case 3: $\alpha>1$. The conditions outlined in Equations D.2 and D.3 are always mutually consistent in this case since the LHS of the condition outlined in Equation D.2 is zero. However, as a result of the convexity of $p_{i}$ at small values of $y_{i}$, it is no longer sufficient to check marginal conditions only.

${ }^{18}$ For few cases, decentralization yields efficiency. Suppose, for example, that $w=m=1, \beta=3 / 4$, and $\rho=7 / 3$. Then Regime 2 from Appendix B and the second bullet in case 1(c) in this appendix apply. Allocations in both scenarios coincide. 
A contest is efficient if $g=g^{*}$ and only high-productivity individuals contribute. To ensure $y_{L}=0$, the utility of low-productivity individuals from not contributing must not fall short of that from starting to do so. That is,

$$
w \cdot m+x^{e}-t_{i}+v_{i}\left(g^{*}\right) \geq w\left(m-y_{i}\right)+x^{e}-t_{i}+p_{i}\left(y_{i}, \mathbf{y}_{-1}^{*}\right) \cdot z+v_{i}\left(g^{*}+r_{L} y_{i}\right)
$$

must hold for all $y_{i} \in\left[0, m-t_{i} / w\right]$ and $i>h$. This condition simplifies to

$$
w y_{i} \geq p_{i}\left(y_{i}, \mathbf{y}_{-i}^{*}\right) z+v_{i}\left(g^{*}+r_{L} y_{i}\right)-v_{i}\left(g^{*}\right) .
$$

As a result of the concavity of $v_{i}$, the RHS of the above inequality is itself smaller or equal to $p_{i}\left(y_{i}, \mathbf{y}_{-i}^{*}\right) z+v_{i}{ }^{\prime}\left(g^{*}\right) r_{L} y_{i}$. Therefore, by Equation 9 a sufficient condition for $y_{i}=0$ to be an optimal choice is

$$
w y_{i}\left(1-\frac{r_{L}}{n r_{H}}\right) \geq p_{i_{z}}
$$

for all $y_{i}>0$. The bracketed term in Equation D.7 is positive. Moreover, for all $i>h, p_{i} \in[0,1)$ because the highproductivity individuals contribute a strictly positive amount. Using the inverse relationship between $\alpha$ and $z$ that makes sure that the high-productivity individuals provide efficiently (defined in Eqn. 22), it follows that Equation D.7 can always be fulfilled if $z \rightarrow 0$. QED.

\section{Appendix E: Proof of Proposition 4}

We will first show that condition outlined in Equation 18 for the highly productive individuals implies the following inverse relationship between decisiveness $\alpha$ and prize $z$ :

$$
z^{o}=\frac{2(1+\beta)}{\alpha}
$$

To see this, recall from Appendix B that the efficient (symmetric) contributions levels amount to $y_{1}=y_{2}=(2+\beta) / 2 w$ and $y_{3}=0$, which gives rise to $g^{*}=r_{H}(2+\beta) / w$. For a Tullock CSF, we can calculate from Equation D. 1 that for an $H$-type individual

$$
\frac{\partial p_{i}\left(y_{i}, \mathbf{y}_{-i}\right)}{\partial y_{i}}=\alpha r_{H}^{\alpha} y_{H}^{\alpha-1} \frac{r_{H}^{\alpha} y_{H}^{\alpha}}{4 r_{H}^{2 \alpha} y_{H}^{2 \alpha}}=\frac{\alpha}{4 y_{H}}
$$

Moreover, for $H$-types,

$$
\sum_{k \neq j} v_{k}{ }^{\prime}\left(g^{*}\right)=\frac{1+\beta}{g^{*}}=w \frac{1+\beta}{r_{H}(2+\beta)}
$$

Plugging these expressions into Equation 18 yields Equation E.1.

However, some $(\alpha, z)$ that satisfy Equation E.1 may fail to solve the sorting problem.

As in Appendix D, Tullock contests with $\alpha<1$ are inefficient because the marginal return at $y_{L}=0$ is infinitely large as long as $r_{L}>0$.

Also the case $\alpha>1$ can be analyzed with a procedure analogous to that in Appendix D. The sufficient condition to deter the low-skilled individual,

$$
w y_{3}\left(1-\frac{\beta r_{L}}{r_{H}(2+\beta)}\right) \geq p_{3} \times z \quad \forall y_{3}>0,
$$

can always be fulfilled for $z \rightarrow 0$.

For $\alpha=1$, Equation E.1 boils down to $z^{o}=2(1+\beta)$. As a result of the concavity of the Tullock function in this case we can restrict our attention to individual 3. At $y_{3}=0$, his marginal utility from (just) contributing,

$$
-w+\beta \frac{r_{L}}{g}+\left.r_{L} \frac{r_{H}\left(y_{1}+y_{2}\right)}{g^{2}} z\right|_{y_{3}=0}
$$

(with $g=r_{H}\left(y_{1}+y_{2}\right)$ ), amounts to

$$
-w+\frac{r_{L}}{g^{*}}(\beta+z)=-w \times\left(1-\frac{\beta+z}{\rho(2+\beta)}\right),
$$


where we set $\rho=r_{H} / r_{L}>1$ and have inserted the efficient levels of $y_{1}, y_{2}$, and $g^{*}=r_{H}\left(y_{1}+y_{2}\right)$. To deter the individual, this expression must be non-positive, which defines an upper bound on the prize $z: z \leq \bar{z}:=(2+\beta) \rho-\beta$. This constraint is compatible with $z=z^{o}$ (from Eqn. E.1) only if

$$
(3-\rho) \beta<2(\rho-1) .
$$

The condition outlined in Equation E.3 always holds if $\rho \geq 3$. If $\rho<3, \beta<[2(\rho-1)] /(3-\rho)$ must hold. QED.

\section{References}

Amegashie, J. Atsu. 2009. American Idol: Should it be a singing contest or a popularity contest? Journal of Cultural Economics 33:265-77.

Amegashie, J. Atsu, and Gordon M. Myers. 2003. Financing public goods via lotteries. Working Paper 2003-1. University of Guelph.

Bergstrom, Theodore, Lawrence Blume, and Hal Varian. 1986. On the private provision of public goods. Journal of Public Economics 29:25-49.

Che, Yeon-Koo, and Ian Gale. 2003. Optimal design of research contests. American Economic Review 93:646-71.

Cornes, Richard, and Todd Sandler. 1984. Easy riders, joint production, and public goods. Economic Journal 94:580-98.

Cornes, Richard, and Todd Sandler. 1994. The comparative static properties of the impure public good model. Journal of Public Economics 54:403-21.

Cornes, Richard, and Todd Sandler. 1996. The Theory of Externalities, Public Goods and Club Goods. Cambridge, UK: Cambridge University Press.

Faravelli, Marco. 2011. The important thing is not (always) winning but taking part: Funding public goods with contests. Journal of Public Economic Theory 13:1-22.

Goeree, Jacob K., Emil Maasland, Sandar Onderstal, and John L. Turner. 2005. How (not) to raise money. Journal of Political Economy 113:897-918.

Lange, Andreas, John A. List, and Michael K. Price. 2007. Using lotteries to finance public goods: Theory and experimental evidence. International Economic Review 48:901-27.

Lazear, Edward P. 1997. Incentives in basic research. Journal of Labor Economics 15:S167-97.

Maeda, Akira. 2008. Optimal lottery design for public financing. Economic Journal 118:1698-1718.

Maurer, Stephen M., and Suzanne Scotchmer. 2004. Procuring knowledge. In Intellectual property and entrepreneurship, edited by Gary D. Libecap. Amsterdam: Elsevier (JAI Press), pp. 1-31.

Morgan, John. 2000. Financing public goods by means of lotteries. Review of Economic Studies 67:761-84.

Skaperdas, Stergios. 1996. Contest success functions. Economic Theory 7:283-90.

Tullock, Gordon. 1980. Efficient rent-seeking. In Toward a theory of the rent-seeking society, edited by James Buchanan, Robert Tollison, and Gordon Tullock. College Station, TX: Texas A\&M University Press, pp. 3-15. 
Reproduced with permission of the copyright owner. Further reproduction prohibited without permission. 